\title{
Domestication De Thunbergia Atacorensis Akoegninou \& Lisowski (Acanthaceae) : Effet Du Type De Substrat Et Du Mode D'eclairement Sur L'aptitude A La Reprise Vegetative Des Boutures Et La Croissance Des Plants
}

\section{Asseh Ebah Estelle}

Doctorant, Université Félix Houphouët Boigny, U.F.R. Biosciences Abidjan.

Laboratoire de Botanique, Institut Botanique Aké -Assi d'Andokoi

(IBAAN), Abidjan, Côte d'Ivoire

\section{Ake-Assi Emma}

Maître Conférences, Université Félix Houphouët Boigny, U.F.R.

Biosciences, Laboratoire de Botanique, Centre National de Floristique Université Félix Houphouët Boigny, Institut Botanique Aké-Assi d'Andokoi (IBAAN), Abidjan, Côte d'Ivoire

\section{Koffi Kouao Jean}

Maitre-assistant, Université Nangui Abrogoua, UFR Sciences de la Nature, Laboratoire de Biologie et Amélioration des Productions Végétales, Institut

Botanique Aké-Assi d'Andokoi (IBAAN)Abidjan, Côte d'Ivoire

\section{Kouassi Akossoua Faustine}

Chargé de Recherches, Université Félix Houphouët Boigny, U.F.R.

Biosciences Centre National de Floristique, Abidjan, Côte d'Ivoire

\section{N'guessan Kouakou Edouard}

Professeur Titulaire, Université Félix Houphouët Boigny, U.F.R.

Biosciences, Laboratoire de Botanique, Abidjan, Côte d'Ivoire

doi: 10.19044/esj.2017.v13n18p328 URL:http://dx.doi.org/10.19044/esj.2017.v13n18p328

\section{Abstract}

We studied the possibility of producing seedlings of Thunbergia atacorensis (Acanthaceae) starting from cuttings under controlled cultivation conditions during 3 months. The objective of this work is to determine the optimal conditions for seedling production Thunbergia atacorensis by vegetative reproduction to allow its extension from professional growers and amateur (consumers). The study consisted to analyze their effect the plant growth, seedlings development, and leaves production. At the end the study, we obtained a rate of 91p.c for vegetative recovery for the cutting in shade 97p.c. for the cutting exposing to sun. However, the cuttings exposed sun 
clearly dissociates cuttings exposed to the shade with a shorter time of recovery. At the end of 3 months, only the sunny seedlings survive despite of the recovery rate of recovery. The study showed that the four environmental factors, the mode of exposure (sun and shade) and time (weeks) have a significant influence on the height and the number of seedlings leaves. The highest seedlings are observed in the shade from weeks 1 to 3. From week 3, only the sunny seedlings continue their growth and their development. The variation of the substrate and the frequency of watering, meanwhile, had no significant influence on the height and number of leaves. In sum, despite of lack of flowering, to produce seedlings of Thunbergia atacorensis, one should initially carry out the propagation by cutting in the shade and then to gradually intensify the light to have well developed seedlings.

Keywords: Thunbergia atacorensis, domestication, cuttings, growth, development

\section{Résumé}

la possibilité de produire des plants de Thunbergia atacorensis (Acanthaceae) a été etudié à partir de boutures dans des conditions de cultures contrôlés pendant 3 mois. L'objectif du présent travail est de déterminer les conditions optimales de production de plants de Thunbergia atacorensis par la reproduction végétative afin de permettre sa vulgarisation auprès des horticulteurs professionnels et amateurs (consommateurs). L'étude a consisté à analyser l'influence de ces variations sur la reprise végétative, le taux de survie des boutures et enfin sur la croissance de dix plants choisis de manière aléatoire au niveau des différentes parcelles. Nous avons obtenu un taux de reprise végétative satisfaisant de 91 p.c. pour les boutures à l'ombre et de 97 p.c. pour les boutures exposées au soleil. Cependant les boutures de parcelles exposées au soleil se démarquent nettement des boutures exposées à l'ombre avec un délai de reprise plus court. Au bout de 3 mois, seuls les plants exposés au soleil survivent malgré le taux de reprise élevé pour toutes les boutures. L'étude de l'influence des paramètres du milieu de culture, indique que le mode d'exposition (ombre et soleil) a une influence significative sur la hauteur et le nombre de feuilles des plants. Les plants les plus hauts sont observés à l'ombre de la $1^{\text {ère }}$ à la $3^{\text {ème }}$ semaine de mesure. À partir de la $3^{\text {ème }}$ semaine, ce sont les plants au soleil qui continuent leur croissance et leur développement. Le type de substrat et la fréquence d'arrosage, n'ont eu aucune influence significative sur la hauteur et le nombre de feuilles des jeunes plants. En somme; malgré le manque de floraison, pour produire des plants de Thunbergia atacorensis; il faudrait, dans un premier temps ; réaliser le bouturage à l'ombre et ensuite intensifier progressivement la lumière pour avoir des plants bien développés. 
Mots-clés : Thunbergia atacorensis, domestication, boutures, croissance, développement

\section{Introduction}

La famille des Acanthaceae fait partie des douze plus grandes familles de plantes à fleurs dans le monde (McDade et al., 2009). C'est une famille qui a une large distribution, et se rencontre généralement en Inde, au Brésil et dans plusieurs pays africains (Avit et al., 1999). Représentée par 221 genres et environ 4000 espèces (Hu et Tsui, 2000), elle est constituée de nombreuses espèces ayant des fleurs très colorées. En Côte d'Ivoire, les Acanthaceae sont classées parmi les familles les plus riches en plantes ornementales (Aké-Assi, 1996). Parmi ces espèces à belles fleurs, une nouvelle espèce, Thunbergia atacorensis (Acanthaceae) a été observée et décrite pour la première fois en 1999 par les professeurs Akoègninou A. et Van Der Maessen, lors de recherches botaniques effectuées au Benin. Par la suite, elle a été introduite en Côte d'Ivoire en 2004. Toutes fois, nous ne disposons d'aucune information dans la littérature signalant sa présence dans un autre pays de l'Afrique de l'ouest; encore moins sur le mode de régénération de la plante. C'est dans ce cadre que notre étude a été initiée. Elle a pour objectif principal de rechercher les conditions optimales et favorables à la régénération de cette plante en Côte d'Ivoire par la voie de la multiplication végétative. De manière plus spécifique, il s'est agit d'étudier l'effet des variations du substrat, de la fréquence d'arrosage, du mode d'exposition à la lumière du soleil, et de leur interaction sur la reprise végétative et la croissance des plants de Thunbergia atacorensis.

\section{Matériel et méthodes}

\section{Matériel végétal}

Le matériel végétal est représenté par des boutures de Thunbergia atacorensis qui est une espèce découverte au Bénin, à l'occasion de recherches botaniques effectuées par les professeurs Akoègninou et Van Der Maessen. Qui ont été gracieusement offerte par le pr Akoègninou de l'université de au paraquou ces boutures ont été repiquée au sein du Centre National de Floristique (C.N.F) (Figure 1)Elle a été rencontrée et observée pour la première fois dans la région de l'Atakora au niveau des galeries forestières (Van der Maessen et Akoègninou, 1999). Outre le Bénin, elle a été introduite en Côte d'Ivoire en 2004, confiée au Professeur Traoré Dossahoua et. Thunbergia atacorensis est une herbe suffrutescente pouvant atteindre 40 à $80 \mathrm{~cm}$ de hauteur, à petite souche ligneuse souterraine. C'est une espèce à tige hispide, à entre-nœud aplatit et profondément sillonnée. Les boutures qui ont servi dans cette étude ont été prélevées sur des pieds 
parents poussant sans soins particuliers au niveau de la parcelle d'expérimentation du Centre National de Floristique (C.N.F.) de l'Université Félix Houphouët Boigny (Côte d'Ivoire). Le matériel technique est constitué d'une ombrière sous laquelle est déposé le bloc de l'ombre et des sachets contenant le substrat, le support des boutures. Deux types de substrats ont été utilisés pour la réalisation des essais. L'un prélevé au C.N.F. est de nature ferralitique (Perraud, 1971) et nommé Sub1. L'autre nommé Sub2 a été prélevé à Bingerville. Ce dernier est de tendance hydromorphe ( Riou, 1961). Ces substrats ont été choisis pour leur ressemblance avec les sols du milieu d'origine de Thunbergia atacorensis.

\section{Matériel végétal}

Le matériel végétal est représenté par des boutures de Thunbergia atacorensis qui est une espèce à tige hispide, à entre-nœud aplatit et profondément sillonnée. Les boutures ont été gracieusement offertes par le Pr Akoègninou de l'Université de Parakou au Benin. Ces boutures ont été repiquées au sein du Centre National de Floristique (C.N.F) de 1'Université Félix Houphouët Boigny (Figure 1).

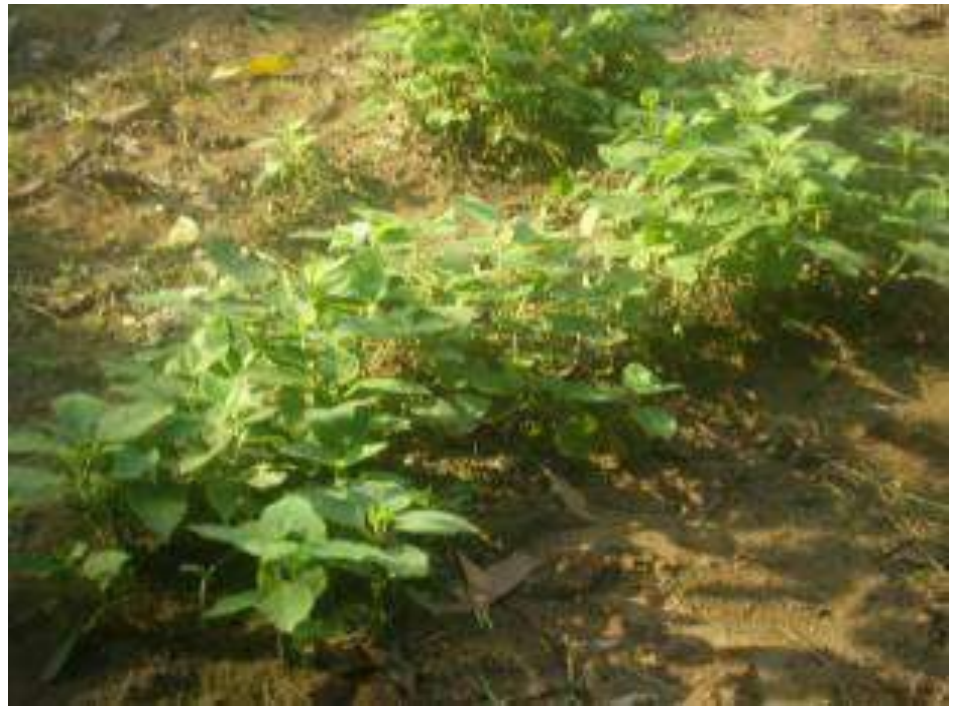

Figure 1: plants de Thunbergia atacorensis (Acanthaceae) en croissance au cnf de l'université felix h

\section{Méthodes}

\section{Préparation des boutures de Thunbergia atacorensis}

L'essai a consisté à produire des plants de Thunbergia atacorensis à partir de boutures. Des individus ont été sélectionnés en tenant compte de l'état physiologique et sanitaire dans lequel ils se trouvaient. Cet état est important pour le prélèvement de la bouture (Dembélé, 2012). Des individus 
sains plus ou moins robustes dont la tige mesurait environ $1 \mathrm{~mm}$ d'épaisseur, n'ayant pas subi l'insolation et portant des feuilles saines et bien développées ont été choisis (Figure 2A). Une portion de la tige de $5 \mathrm{~cm}$ à $10 \mathrm{~cm}$ est prélevée (Figure 2B ; 2C) par une section nette à l'aide d'un sécateur en dessous d'un nœud (Rosenn et al., 2004). La bouture (Figure 2D) obtenue porte au moins deux nœuds car c'est de ces derniers que les repousses recherchées vont démarrer. Ainsi, sur chaque pied mère, 3 portions de tiges ont été sectionnées. Une fois prélevé, les boutures à utiliser ont été habillées. L'habillage des boutures (Figure 2D) est nécessaire à la réussite du bouturage. Cela s'est fait par élimination des feuilles insérées au niveau des nœuds pour éviter l'évapotranspiration des boutures (Kalingani et al., 2007). La suppression des feuilles se fait en évitant d'abîmer les bourgeons à l'aisselle des feuilles. Les boutures prélevées, ont été protégées pour éviter leur dessiccation (Dorion, 2013). Pour ce faire, la base des boutures a été recouverte avec du papier journal légèrement mouillé et maintenu avec le fil à tresse (Figure 2E). Ensuite elles ont été tenues à l'abri du soleil dans un endroit sec avant leur mise en terre.

\section{Préparation des boutures de Thunbergia atacorensis}

Des plants ont été sélectionnés en tenant compte de leur état physiologique et sanitaire. Cet état est important pour le prélèvement de la bouture (Dembélé, 2012). Ces plants sains ont une tige qui mesurait environ $1 \mathrm{~mm}$ d'épaisseur. Ils n'ont pas subi l'insolation et portent des feuilles saines et bien développées (Figure 2A). Une portion de la tige de $5 \mathrm{~cm}$ à $10 \mathrm{~cm}$ est prélevée (Figure 2B ; 2C) par une section nette à l'aide d'un sécateur en dessous d'un nœud (Rosenn et al., 2004). La bouture (Figure 2D) obtenue porte au moins deux nœuds car c'est de ces derniers que les repousses recherchées vont démarrer. Ainsi, sur chaque pied mère, 3 portions de tiges ont été sectionnées. Une fois prélevé, les boutures à utiliser ont été habillées. L'habillage des boutures (Figure 2D) est nécessaire à la réussite du bouturage. Cela s'est fait par élimination des feuilles insérées au niveau des nœuds pour éviter l'évapotranspiration des boutures (Kalingani et al., 2007). La suppression des feuilles se fait en évitant d'abîmer les bourgeons à l'aisselle des feuilles. Les boutures prélevées, ont été protégées pour éviter leur dessiccation (Dorion, 2013). Pour ce faire, la base des boutures a été recouverte avec du papier journal légèrement mouillé et maintenu avec le fil à tresse (Figure 2E). Ensuite elles ont été tenues à l'abri du soleil dans un endroit sec avant leur mise en terre. 


\section{Description des essais \\ Dispositif expérimental}

L'essai a été réalisé, en vue d'étudier l'influence des facteurs environnementaux et leur interaction sur la croissance et le développement des plants. Les paramètres étudiés sont le mode d'éclairement, le type de substrat et la fréquence d'arrosage. Le mode d'éclairement a été testé à partir de deux modalités : l'exposition à l'ombre et au soleil. Le type de substrat présente également deux modalités : Sub1 (substrat prélevé au C.N.F.) et Sub2 (substrat prélevé à Bingerville). La fréquence d'arrosage ; Ar1 (chaque semaine) et Ar2 (tous les trois jours). L'effet de ces paramètres a été suivi pendant sept semaines. Ce qui fait du temps un paramètre supplémentaire avec sept modalités (semaine 1 à semaine 7). En tenant compte du nombre des paramètres considérés et de l'objectif de l'étude, le dispositif expérimental utilisé est le criss-cross appelé encore cross over. Ce dispositif permet d'étudier l'influence et l'interaction de plusieurs paramètres sur l'expression d'une ou plusieurs grandeurs chez un ou plusieurs individus (Mondegnon ; 2012). Le principe est de définir des blocs qui sont divisés en parcelles au nombre des paramètres étudiés. Ensuite ces parcelles sont divisées en sous parcelles ou placettes. En effet, 2 grands blocs ont été retenus, un bloc à l'ombre et un autre au soleil (Figure 3). Au niveau de chaque bloc, nous avons défini 4 parcelles. Ensuite, chaque parcelle a été subdivisée en 3 sous parcelles donnant une disposition en colonne (Figure 3). Dans chaque placette, nous avons repiqué 10 boutures. Ainsi, chaque parcelle comprend 30 boutures et chaque grand bloc 120 boutures. Pour tout le dispositif, nous avons 240 boutures. Les dimensions de chaque bloc sont 2,5 m x $5 \mathrm{~m}$. celles des parcelles $1 \mathrm{~m}$ x 2,5 m et celles des placettes $1 \mathrm{~m}$ x 0,8 $\mathrm{m}$.

\section{Dispositif expérimental}

Les facteurs étudiés sont le mode d'éclairement, le type de substrat et la fréquence d'arrosage. Le mode d'éclairement a été testé à partir de deux modalités : l'exposition à l'ombre et au soleil. Le type de substrat présente également deux modalités : Sub1 (substrat prélevé au C.N.F.) et Sub2 (substrat prélevé à Bingerville). La fréquence d'arrosage; Ar1 (chaque semaine) et Ar2 (tous les trois jours). Ainsi, en tenant compte des modalités, nous avons huit traitements qui ont été effectués (OmbSub1Ar1; OmbSub1Ar2 ; OmbSub2Ar1; OmbSub2Ar2 ; SolSub1Ar1 ; SolSub1Ar2 ; SolSub2Ar1; SolSub2Ar1). L'effet de ces traitements a été suivi pendant sept semaines. Le dispositif expérimental utilisé est le criss-cross appelé encore cross over. Ce dispositif permet d'étudier l'influence et l'interaction de plusieurs paramètres sur l'expression d'une ou plusieurs grandeurs chez un ou plusieurs individus (Mondegnon ; 2012). Le principe est de définir des 
blocs qui sont divisés en parcelles au nombre des facteurs étudiés. Ensuite ces parcelles sont divisées en sous parcelles ou placettes. En effet, 2 grands blocs ont été retenus, un bloc à l'ombre et un autre au soleil (Figure 3). Au niveau de chaque bloc, nous avons défini 4 parcelles. Ensuite, chaque parcelle a été subdivisée en 3 sous parcelles donnant une disposition en colonne (Figure 3). Dans chaque placette, nous avons repiqué 10 boutures. Ainsi, chaque parcelle comprend 30 boutures et chaque grand bloc 120 boutures. Pour tout le dispositif, nous avons 240 boutures. Les dimensions de chaque bloc sont $2,5 \mathrm{~m} \times 5 \mathrm{~m}$. Celles des parcelles sont de $1 \mathrm{~m}$ x 2,5 m et celles des placettes sont de $1 \mathrm{~m}$ x $0,8 \mathrm{~m}$.
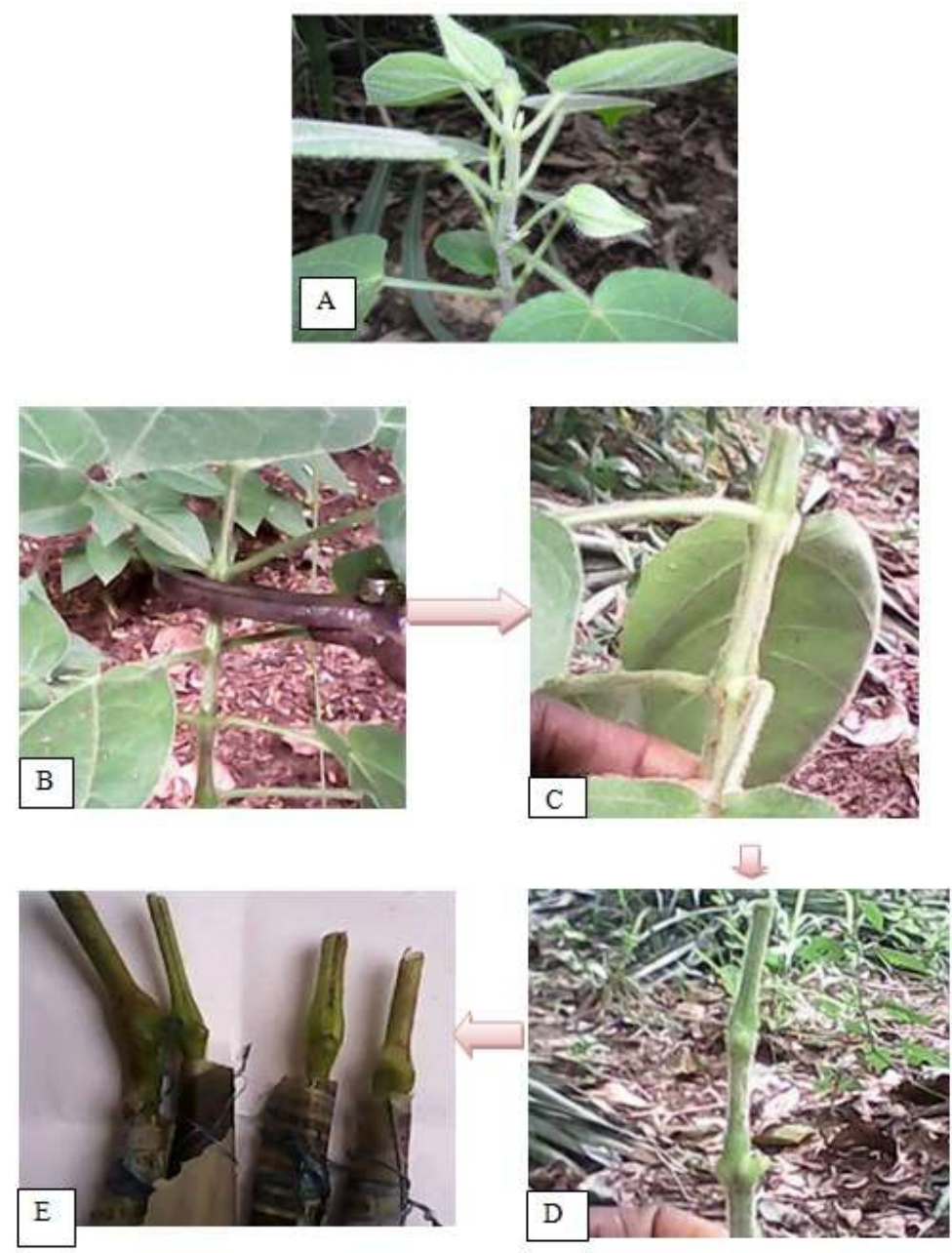

Figure 2 : Étapes de préparation de boutures de Thunbergia atacorensis

A : Aperçu d'un plant ; B : prélèvement de bouture ; C : bouture prélevée ; D : bouture habillée ; E : conservation de boutures. 
qu'en est-il de A, B et C ????

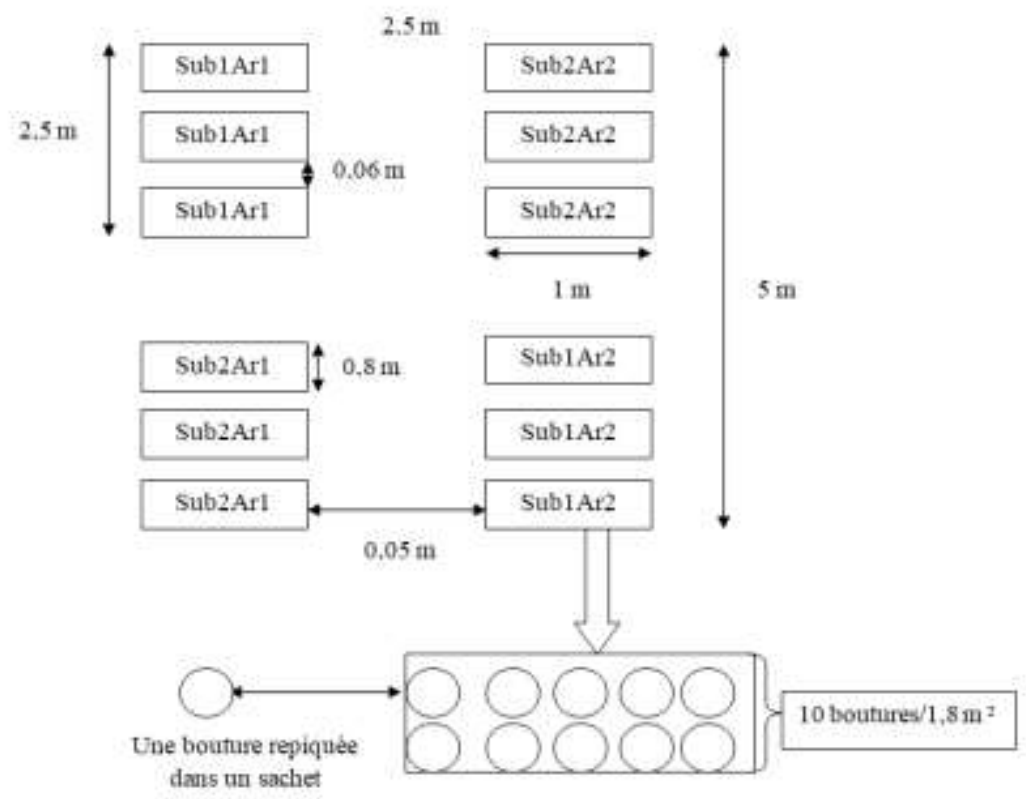

Figure 3 : Schéma du dispositif expérimental

\section{Mise en terre des boutures}

Après avoir fait un trou dans le substrat, les boutures prélevées et habillées ont été enfoncées verticalement jusqu'en dessous du deuxième nœud (Figure 4I ; 4J). Ce mode de repiquage a été décrit et utilisé pour le bouturage de nombreuses espèces ornementales (Rosenn et al., 2004). Après la mise en terre, une légère pression a été exercée autour de la bouture (Figure 4K). Cette pression permet de tasser et de consolider la terre autour de la bouture. Ensuite, toutes les boutures repiquées ont été arrosées de deux manières : soit une fois par semaine ou tous les trois jours, et ce, jusqu'à la fin de notre étude. Enfin, pour éviter que les boutures et les jeunes plants ne soient attaqués par les insectes, les escargots et autres gastéropodes, nous avons pulvérisé l'insecticide Cypercalso et le Caldehyde autour des boutures. 

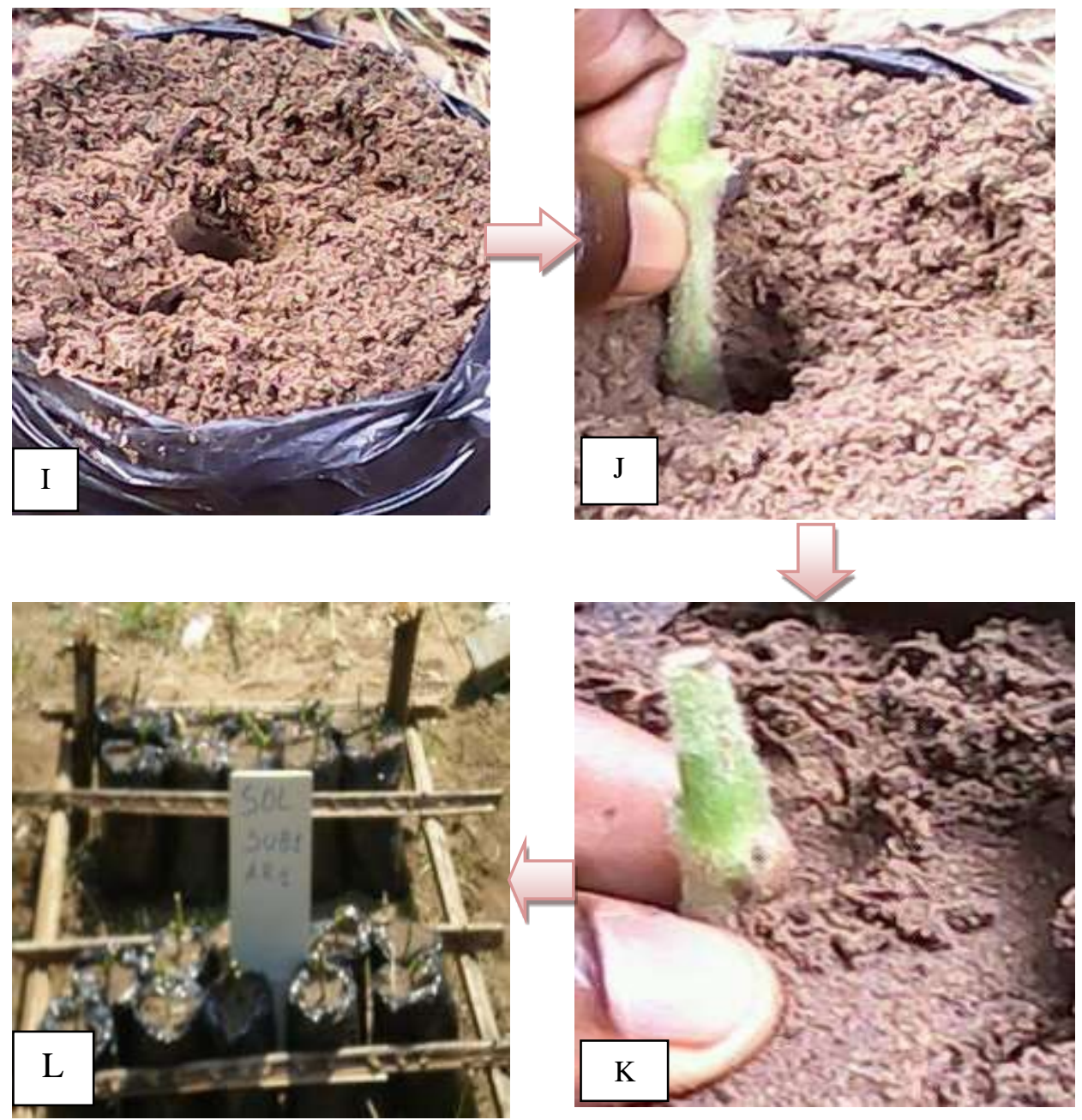

Figure 4 : Étapes de mise en terre des boutures.

$\mathrm{I}$; $\mathrm{J}$ : mise en terre d'une bouture ; $\mathrm{K}$ : consolidation du substrat autour de la bouture ; $\mathrm{L}$ : vue d'ensemble d'une parcelle après la mise en terre des boutures

\section{Relevés et Analyse de données Paramètres mesurés}

Après la mise en terre des boutures, nous avons observé la capacité des boutures à se régénérer. Pour ce faire, nous avons compté les boutures portant des bourgeons débourrés. Cette opération s'est déroulée sur 11 jours et l'observation s'est faite à intervalle de 3 jours. 21 jours après la mise en terre, nous avons sélectionné de manière aléatoire 10 plants dans chaque parcelle unitaire. Sur ces plants, nous avons évalué les paramètres de croissance. La hauteur des plants a été mesurée à l'aide d'un mètre ruban. La mesure consistait à tendre verticalement le mètre, entre le collet et la base des feuilles nouvellement formées. Les feuilles ont été comptées également 
chaque semaine, à partir du premier nœud d'insertion jusqu'aux feuilles apicales.

\section{Analyse des données}

\section{Taux de reprise végétative}

Le taux de reprise végétative (Tr): est obtenu en faisant le rapport entre le nombre de boutures ayant repris, c'est-à-dire les boutures portant des bourgeons débourrés (Nr) par le nombre de boutures repiquées initialement (Nt). La formule est la suivante :

$$
\mathrm{Tr}=(\mathrm{Nr} / \mathrm{Nt}) \times 100
$$

\section{Taux de survie des plants}

Le taux de survie des plants (Ts) s'obtient en faisant le rapport entre le nombre de plants ayant survécu $(\mathrm{Pv})$ et le nombre de boutures initialement repiquées (Nt), le tout multiplié par 100 (Bekker et al. ,2004). Dans notre cas, ce paramètre a été pris en compte 3 mois après la mise en terre des boutures et est déterminé selon la formule ci-dessous :

$$
\text { Ts }=(\mathbf{P v} / \mathrm{Nt}) \times 100
$$

\section{Hauteur moyenne des plants}

hauteur moyenne des plants $(\mathrm{Hm})$ est le rapport de la hauteur totale $(\mathrm{Ht}) \mathrm{des}$ plants sur le nombre de plants choisis (Ns) par parcelle unitaire. Elle a été calculée sur le temps total de l'essai selon la formule ci-après:

$$
\mathrm{Hm}=\mathrm{Ht} / \mathrm{Ns}
$$

\section{Nombre moyen de feuilles des plants}

nombre moyen de feuilles par plant (Fm) s'obtient en faisant le rapport du nombre total de feuilles (Ft) et du nombre de plants (Ns) choisis par parcelle unitaire. Il se calcule de la manière suivante :

$$
\mathbf{F m}=\mathbf{F t} / \mathrm{Ns}
$$

\section{Analyses statistiques}

Les différences entre les hauteurs moyennes, le nombre moyen de feuilles des plants, ont été évaluées à travers une analyse de variance ANOVA à plusieurs facteurs avec mesures répétées. C'est une méthode d'analyse qui permet de percevoir l'évolution au cours du temps d'une grandeur en fonction de différents paramètres (Myriam, 2008). Le principe de base de ce test est de voir si la variabilité des observations est fonction des facteurs étudiés. Lorsqu'une différence est trouvée, les moyennes sont classées à 
l'aide du test de Tukey au seuil de 0,05. Pour la réalisation de ces tests statistiques, le logiciel STATISTICA.7 a été utilisé.

\section{Résultats}

\section{Taux de reprise végétative et de survie des plants de Thunbergia atacorensis}

Les courbes du taux de reprise végétative présentent en général trois phases pour tous les traitements (Figure 5). Une première phase, caractérisée par une pente nulle, qui dure 5 jours pour les parcelles à l'ombre et 3 jours pour les parcelles exposées au soleil. Ensuite une seconde phase, traduisant l'évolution graduelle du taux de reprise végétative, atteint un pic au neuvième jour. Enfin une troisième phase caractérisée par une pente descendante de la courbe à partir du neuvième jour. Au 11 ème jour, nous avons 90 à $93 \%$ de taux de reprise végétative en moyenne pour les boutures exposées à l'ombre et atteint environ $100 \%$ pour celles exposées au soleil (Figure 6). 10 semaines après la mise en terre des boutures, un taux de survie de $0,00 \%$ est obtenu pour les plants des parcelles à l'ombre et plus de $90 \%$ pour les plants des parcelles au soleil, (Figure 7).Au 11 ème jour et les autres jours ???, nous avons noté 91.p.c. de taux de reprise végétative en moyenne pour les boutures exposées à l'ombre et 97.p.c. pour celles exposées au soleil (Figure 5). Nous avons obtenu un taux de survie de 0,00.p.c. pour les plants des parcelles à l'ombre et de plus de 90.p.c pour les plants des parcelles au soleil, 10 semaines après la mise en terre des boutures (Figure 6). Revoir l'ordre des figures

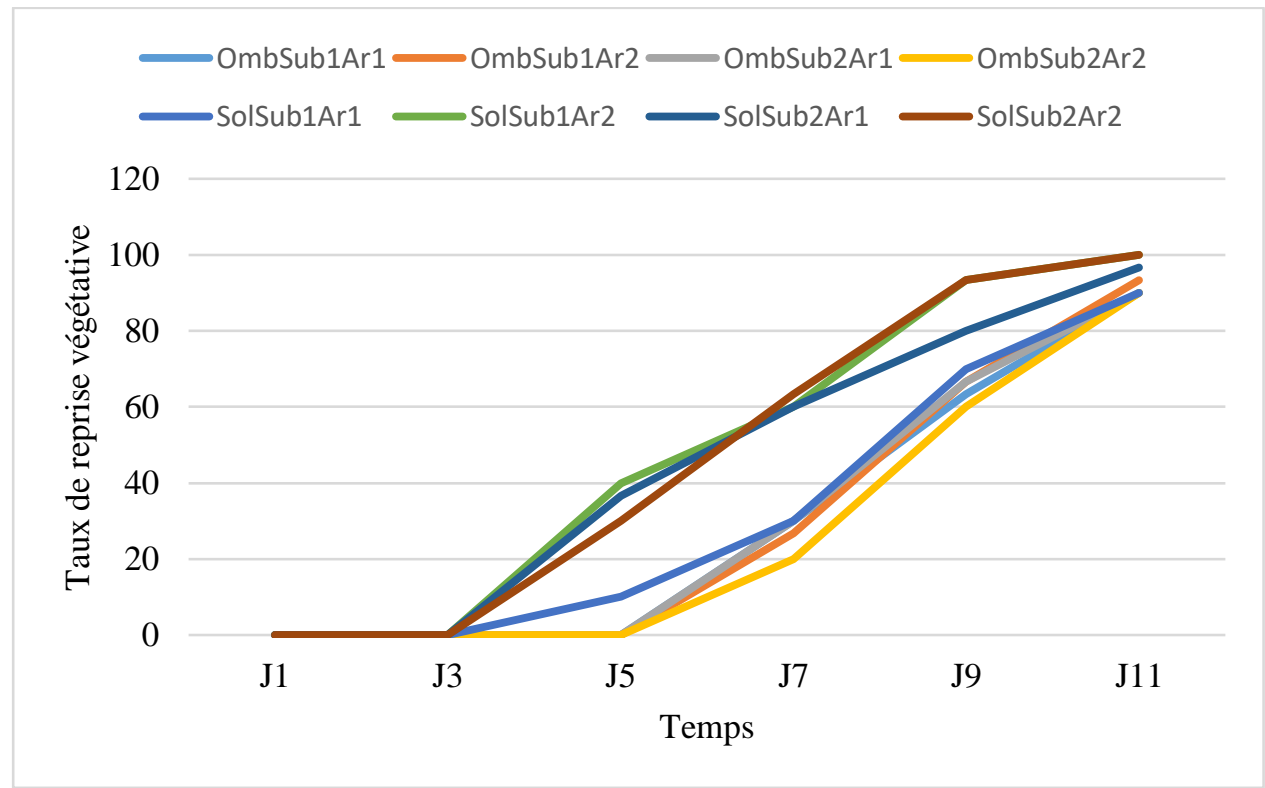

Figure 5 : Evolution des taux de reprise végétative des boutures de Thunbergia atacorensis en fonction du temps 


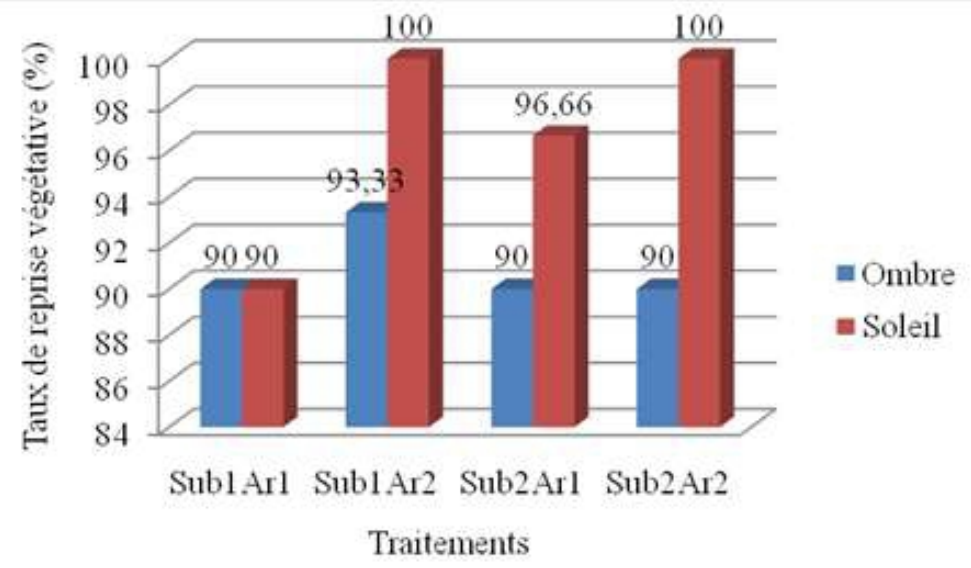

Figure 6 : Taux de reprise végétative des boutures au 11ème jour après la mise en terre desboutures

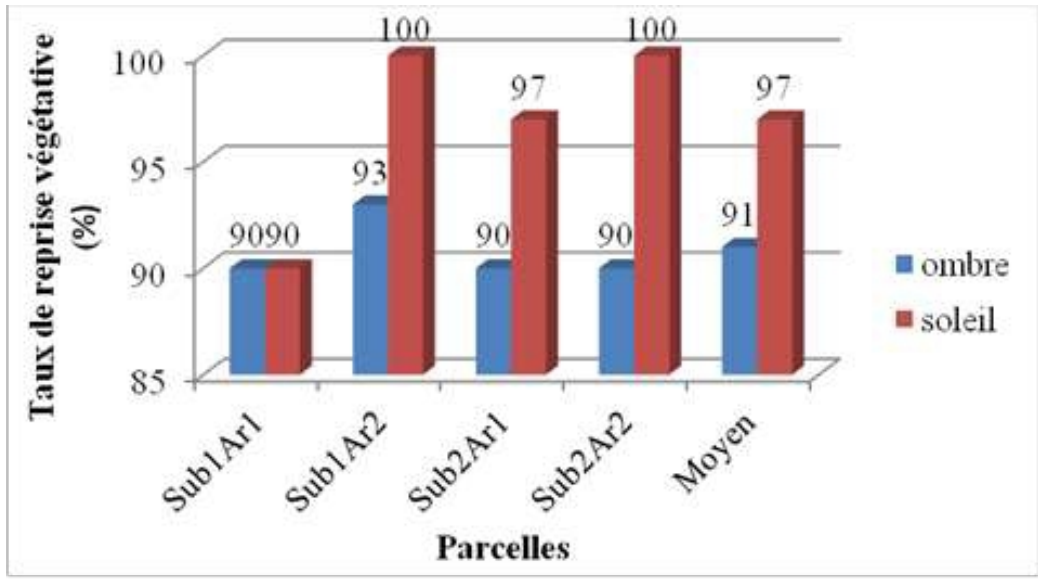

Figure 5 : Taux de reprise végétative des boutures 11 après la mise en terre

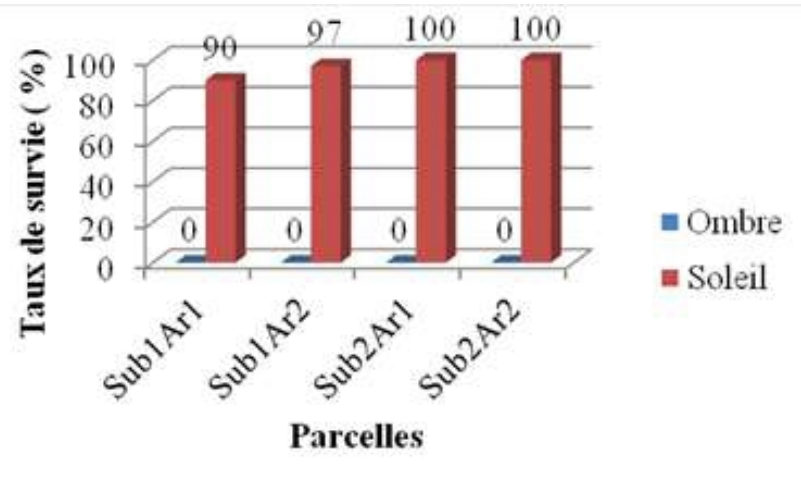

Figure 6 : Taux de survie des plants 10 semaines après la mise en terre 


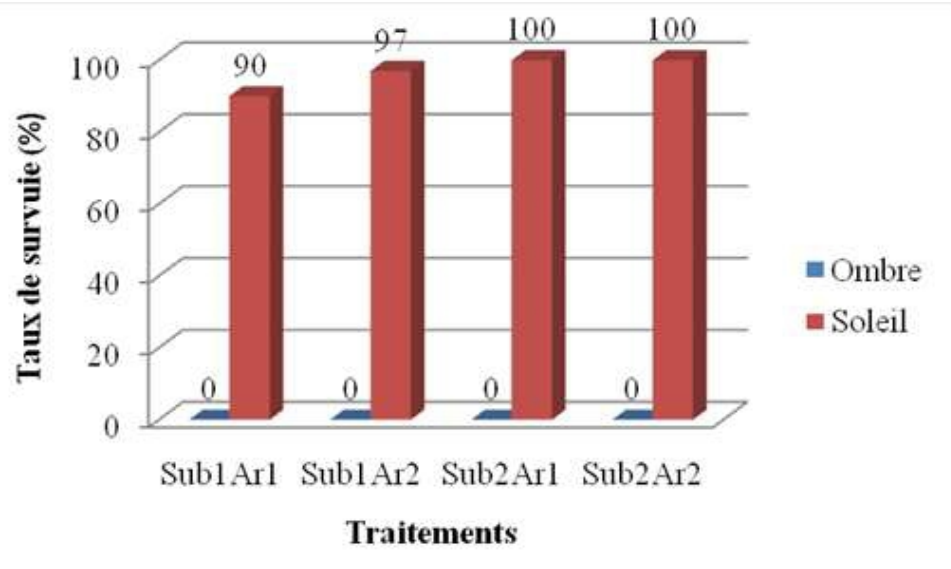

Figure 7 : Taux de survie des plants 10 semaines après la mise en terre des boutures

\section{Incidence des paramètres étudiés sur la hauteur moyenne des plants}

Les hauteurs moyennes des plants issus des boutures repiquées dans les substrats Sub1 et Sub2 sont respectivement 3,02 $\pm 2,91 \mathrm{~cm}$ et 3,36 $\pm 2,94$ $\mathrm{cm}$ (Tableau I). L'analyse de variance révèle que, d'un type de substrat à l'autre, il n'y a pas de différence significative entre les hauteurs moyennes des plants. Les hauteurs moyennes des plants sont 3,03 $\pm 2,93 \mathrm{~cm}$ et 3,35 \pm $2,92 \mathrm{~cm}$ respectivement pour les fréquences d'arrosage Ar1 et Ar2. L'analyse de variance montre que les hauteurs moyennes des plants sont statistiquement identiques quelque soit la fréquence d'arrosage (Tableau I). Les moyennes affectées de la même lettre sont statistiquement identiques pour le test de Tukey au seuil 0,05 ; Expositions $(F=33,21 ; p=0,000)$; Temps $(F=21,86 p=0,000)$. En ce qui concerne le mode d'exposition, les hauteurs moyennes des plants sont 3,71 $\pm 3,87 \mathrm{~cm}$ et 2,66 $\pm 1,29 \mathrm{~cm}$ respectivement pour les plants des parcelles exposées à l'ombre et au soleil. La comparaison de la hauteur moyenne des plants montre qu'il existe une différence significative entre les moyennes $(F=33,21 ; p=0,000)$. Les plants issus des parcelles exposées à l'ombre sont plus hauts que les plants exposés au soleil. Au niveau du temps, la hauteur moyenne des plants pour la semaine $\mathrm{S} 1$ est de $3,32 \pm 1,98 \mathrm{~cm}$. Cette hauteur augmente progressivement de $\mathrm{S} 1$ à $\mathrm{S} 4$ en passant de 3,32 $\pm 1,98 \mathrm{~cm}$ à $4,64 \pm 3,21 \mathrm{~cm}$. À partir de $\mathrm{S} 5$, la hauteur moyenne baisse progressivement et passe de 2,53 $\pm 3,07 \mathrm{~cm}$ à 1,89 \pm $2,25 \mathrm{~cm}$ pour S7 (Tableau I). L'analyse de variance montre qu'il existe des différences significatives entre les hauteurs moyennes des plants $(F=21,86$; $p=0,000)$.

Tableau I : Comparaison de la hauteur moyenne des plants en fonction des paramètres étudiés

\begin{tabular}{ccc}
\hline Paramètres & modalités & Hauteur moyenne $(\mathrm{cm})$ \\
\hline Type de Substrat & Sub1 & $3,02 \pm 2,91 \mathrm{a}$ \\
\hline
\end{tabular}




\begin{tabular}{ccc}
\hline & Sub2 & $3,36 \pm 2,94 \mathrm{a}$ \\
\hline \multirow{2}{*}{ Type d'Arrosages } & Ar1 & $3,03 \pm 2,93 \mathrm{a}$ \\
\cline { 2 - 3 } & Ar2 & $3,35 \pm 2,92 \mathrm{a}$ \\
\hline \multirow{2}{*}{ Expositions } & Ombre & $3,71 \pm 3,87 \mathrm{a}$ \\
\cline { 2 - 3 } & Soleil & $2,66 \pm 1,29 \mathrm{~b}$ \\
\hline \multirow{5}{*}{ Temps } & $\mathrm{S} 1$ & $3,32 \pm 1,98 \mathrm{bc}$ \\
\cline { 2 - 3 } & $\mathrm{S} 2$ & $4,27 \pm 3,21 \mathrm{~cd}$ \\
\cline { 2 - 3 } & $\mathrm{S} 3$ & $4,64 \pm 3,21 \mathrm{c}$ \\
\cline { 2 - 3 } & $\mathrm{S} 4$ & $4,64 \pm 3,21 \mathrm{c}$ \\
\cline { 2 - 3 } & $\mathrm{S} 5$ & $2,53 \pm 3,07 \mathrm{ab}$ \\
\cline { 2 - 3 } & $\mathrm{S} 6$ & $1,86 \pm 2,27 \mathrm{a}$ \\
\hline
\end{tabular}

Pour chacun des paramètres à savoir, hauteur ou nombre de feuille, suivre l'évolution des valeurs de la combinaison substratarrosage-éclairement en fonction du temps. Cela revient assez lourd. Je suggère pour chaque paramètre mesuré, fixer le temps de mesure soit, sept semaines après la mise en terre. Cela revient à dire : pour un type de substrat, une fréquence d'arrosage et un mode d'éclairement, quelle a été la hauteur ou le nombre de feuilles des plants après $\mathbf{7}$ semaines de croissance

\section{Incidence des facteurs étudiés sur la hauteur moyenne des plants}

Les hauteurs moyennes des plants issus des boutures repiquées en fonction des traitements appliqués varient de $22.07 \pm 5.02 \mathrm{~cm}$ à $35.5 \pm$ $24.93 \mathrm{~cm}$. (Tableau I). La comparaison des moyennes montre que la hauteur moyenne des plants à l'ombre est plus élevée que celle des plants exposés au soleil. L'analyse de variance révèle que, d'un type de traitement à l'autre, il n'y a pas de différence significative entre les hauteurs moyennes des plants. La hauteur moyenne des plants est évolutive tout au long des 7 semaines de mesure (figure 8). Pour les traitements OmbSub1Ar1; OmbSub1Ar2 ; OmbSub2Ar1 et OmbSub2Ar2, elle augmente rapidement jusqu'à atteindre le pic de croissance respectivement les $3^{\text {ème }}, 4^{\text {ème }}, 2^{\text {ème }}$ et $3^{\text {ème }}$ semaines de mesure pour ensuite chuter progressivement jusqu'à la mort total des plants. Par contre, la hauteur moyenne des plants des traitements SolSub1Ar1; SolSub1Ar2; SolSub2Ar1 et SolSub2Ar2 augmente progressivement pou atteindre une valeur plus élevée à la $7^{\text {ème }}$ semaine de mesure.

Tableau I : Comparaison de la hauteur moyenne des plants en fonction des traitements étudiés 10 semaines après la mise en terre

\begin{tabular}{ccc}
\hline & \multicolumn{2}{c}{ Hauteurs moyennes des plants } \\
\cline { 2 - 3 } Traitements & Ombre & Soleil \\
\hline Sub1Ar1 & $26,5 \pm 29,25 \mathrm{a}$ & $22,07 \pm 5,02 \mathrm{a}$ \\
\hline Sub1Ar2 & $35,5 \pm 24,93 \mathrm{a}$ & $22,5 \pm 6,13 \mathrm{a}$ \\
\hline Sub2Ar1 & $33,00 \pm 25,91 \mathrm{a}$ & $27,85 \pm 7,28 \mathrm{a}$ \\
\hline
\end{tabular}


Sub2Ar2

$33,57 \pm 29,84 \mathrm{a}$

$27,00 \pm 8,66 \mathrm{a}$

Les moyennes affectées de la même lettre sont statistiquement identiques pour le test de Tukey au seuil $0,05(F=27,19 ; p=0,001)$

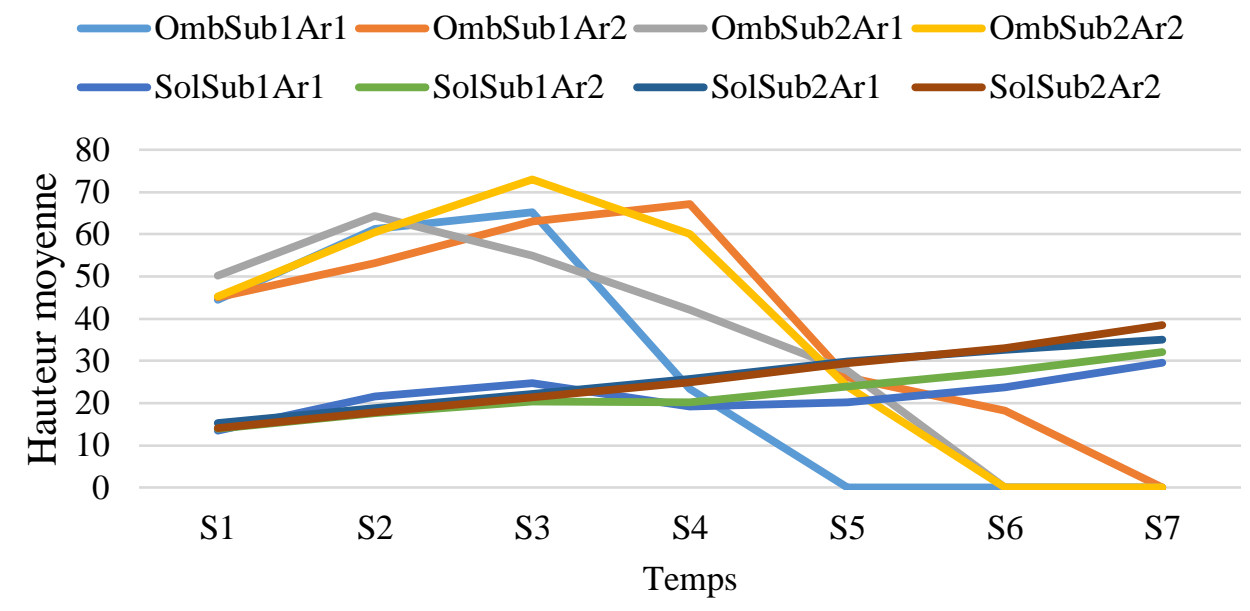

Figure 8: Variation de la hauteur moyenne des plants en fonction du temps

\section{Incidence des paramètres étudiés sur le nombre moyen de feuilles des plants}

Les plants cultivés sur les substrats Sub1 et Sub2 ont développé respectivement en moyenne 4,28 $\pm 3,22$ et 4,90 \pm 3,35 feuilles (Tableau II). L'analyse de variance ne révèle aucune différence significative entre le nombre moyen de feuilles des plants. En fonction des fréquences d'arrosage (Tableau II), les plants ont développé en moyenne 4,42 $\pm 3,35$ et 4,77 $\pm 3,04$ feuilles respectivement pour les fréquences d'arrosage Ar1 et Ar2. L'analyse de variance révèle que quelque soit la fréquence d'arrosage le nombre moyen de feuilles est statistiquement identique pour tous les plants. Le nombre moyen de feuilles par plant est de 3,15 $\pm 3,87$ et 6,03 $\pm 3,36$ respectivement pour les plants issus des parcelles exposées à l'ombre et au soleil (Tableau II). L'analyse de variance indique des différences significatives entre le nombre moyen de feuilles des plants $(F=22,16 ; p=0,000)$. Le premier groupe est constitué des plants exposés au soleil qui ont donné le plus grand nombre de feuilles. Quant au deuxième groupe, ceux sont les plants exposés à l'ombre présentent le plus faible nombre moyen de feuilles. Le nombre moyen de feuilles des plants pour la semaine $\mathrm{S} 1$ est de $4,40 \pm 1,32$. Ce nombre augmente progressivement de la semaine $S 1$ à la $S 3$ et passe de 4,40 $\pm 1,32$ à $5,87 \pm 2,07$. Â partir de $\mathrm{S} 4$ le nombre de feuilles baisse et passe de $4,95 \pm 3,24$ à 4,07 $\pm 3,79$ au niveau de S7 (Tableau II). L'analyse de variance 
montre des différences significatives entre le nombre moyen de feuilles des plants pour les sept semaines de mesures $(F=21,86 ; p=0,000)$. La comparaison des moyennes de feuilles révèle trois groupes. Pour le premier groupe, les plants présentent le plus grand nombre de feuilles et ceux à partir de S3. Les plus faibles nombres de feuilles sont observé à partir des semaines $\mathrm{S} 5$; S6 et $\mathrm{S} 7$ pour le deuxième groupe. Enfin, le troisième groupe présente des valeurs intermédiaires aux deux extrémités et sont observées pour les semaines $\mathrm{S} 1$ et $\mathrm{S} 2$.

Les moyennes affectées de la même lettre sont statistiquement identiques pour le test de Tukey au seuil 0,05 ; Expositions $(F=22,16 ; p=0,00)$; Temps $(F=21,86 ; p=0,000)$.

Tableau II : Comparaison du nombre moyen de feuilles des plants en fonction des paramètres étudiés. Même remarque

\begin{tabular}{|c|c|c|}
\hline Paramètres & modalités & Nombre feuilles moyen \\
\hline \multirow{2}{*}{ Type de Substrat } & Sub1 & $4,28 \pm 3,22 \mathrm{a}$ \\
\hline & Sub2 & $4,90 \pm 3,16 a$ \\
\hline \multirow{2}{*}{ Type d'Arrosage } & Ar1 & $4,42 \pm 3,35 a$ \\
\hline & Ar2 & $4,77 \pm 3,04 \mathrm{a}$ \\
\hline \multirow{2}{*}{ Expositions } & Ombre & $3,15 \pm 3,87 a$ \\
\hline & Soleil & $6,03 \pm 3,36 b$ \\
\hline \multirow{7}{*}{ Temps } & S1 & $4,40 \pm 1,32 \mathrm{abc}$ \\
\hline & S2 & $5,27 \pm 1,32 \mathrm{~cd}$ \\
\hline & S3 & $5,87 \pm 2,07 d$ \\
\hline & S4 & $4,95 \pm 3,24 \mathrm{bcd}$ \\
\hline & S5 & $4,07 \pm 3,79 \mathrm{ab}$ \\
\hline & S6 & $4,07 \pm 3,79 \mathrm{ab}$ \\
\hline & S7 & $4,07 \pm 3,79 \mathrm{ab}$ \\
\hline
\end{tabular}

Incidence des facteurs étudiés sur le nombre moyen de feuilles des plants

Le nombre moyen de feuilles varie de 21,54 $\pm 22,53$ à 60,71 $\pm 12,15$ (tableau 2). L'analyse de variance montre des différences significatives $(F=$ 27,18; $p=0,001$ ) entre le nombre moyen de feuilles des plants pour les différents traitements. La comparaison des moyennes de feuilles révèle trois groupes. Pour le premier groupe, les plants présentent le plus faible nombre de feuilles et sont observés pour le traitement OmbSub1Ar1 (21,54 \pm 22,53). Le plus grand nombre de feuilles observé pour le traitement SolSub2Ar1 $(60,71 \pm 12,15)$ constitue le deuxième groupe. Enfin, le troisième groupe présente des valeurs intermédiaires aux deux extrémités et sont observées pour les traitements OmbSub1Ar2; OmbSub2Ar1; OmbSub2Ar2 ; SolSub1Ar2 ; SolSub2Ar2. En général, Le test de Tukey au seuil 0,05 révèle que les plants exposés au soleil portent en moyenne plus de feuilles que les plants exposés à l'ombre. Le nombre de moyen de feuilles varie en fonction du temps et on observe qu'il est généralement plus élevé pour les plants des traitements SolSub1Ar1, SolSub1Ar2, SolSub2Ar1 et 
SolSub2Ar2 que les plants des traitements OmbSub1Ar1, OmbSub1Ar2, OmbSub2Ar1 et OmbSub2Ar2 (Figure 9).

Tableau II : Comparaison du nombre moyen de feuilles des plants en fonction des traitements étudiés pendant les 7 semaines de mesure

\begin{tabular}{ccc}
\hline & \multicolumn{2}{c}{ Hauteurs moyennes des plants } \\
\cline { 2 - 3 } Traitements & Ombre & Soleil \\
\hline Sub1Ar1 & $21,54 \pm 22,53 \mathrm{a}$ & $47,45 \pm 7,27 \mathrm{ab}$ \\
\hline Sub1Ar2 & $33,25 \pm 22,10 \mathrm{ab}$ & $51,17 \pm 7,36 \mathrm{ab}$ \\
\hline Sub2Ar1 & $30,71 \pm 22,23 \mathrm{ab}$ & $60,71 \pm 12,15 \mathrm{~b}$ \\
\hline Sub2Ar2 & $31,25 \pm 24,58 \mathrm{ab}$ & $57,68 \pm 14,00 \mathrm{ab}$ \\
\hline
\end{tabular}

Les moyennes affectées de la même lettre sont statistiquement identiques pour le test de Tukey au seuil 0,05 $(F=27,18 ; p=0,001)$

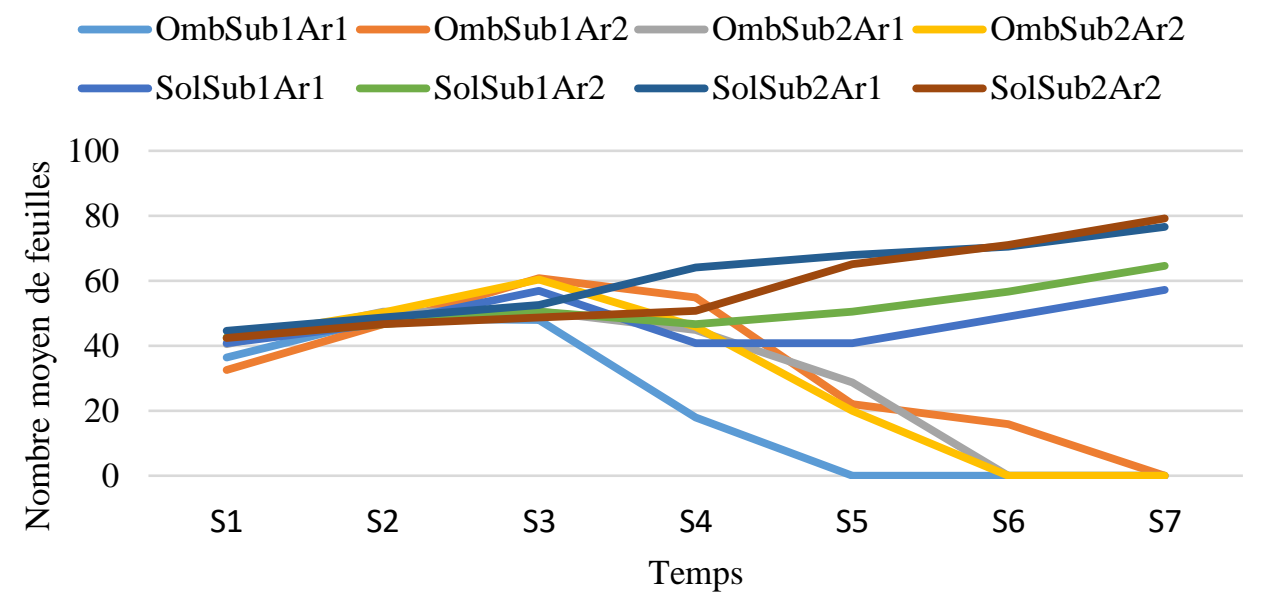

Figure 9: Variation du nombre moyen de feuilles des plants en fonction du temps

\section{Incidence de l'interaction des paramètres étudiés sur la hauteur moyenne des plants}

Lorsqu'on considère l'interaction Substrats-Arrosages (Tableau III), les hauteurs moyennes des plants varient de 2,70 $\pm 2,97 \mathrm{~cm}$ à $3,36 \pm 3,02 \mathrm{~cm}$ respectivement pour les parcelles Sub1Ar1; Sub1Ar2; Sub2Ar1 et Sub2Ar2. L'analyse de variance révèle qu'il n'y a aucune différence significative entre les hauteurs moyennes des plants. Les hauteurs moyennes des plants en fonction de l'association Expositions-Substrats varient de 3,54 $\pm 3,79 \mathrm{~cm}$ à $2,83 \pm 1,10 \mathrm{~cm}$ respectivement pour les parcelles Sub1-omb; Sub1-sol; Sub2-omb et Sub2-sol (Tableau III). L'analyse de variance montre que les hauteurs moyennes des plants sont statistiquement identiques. Au niveau de l'effet combiné Arrosages-Expositions, les hauteurs moyennes des plants issus des associations Ar1-omb; Ar1-sol; Ar2-omb et Ar2-sol 
respectivement varient de 3,41 $\pm 3,88 \mathrm{~cm}$ à 2,68 $\pm 1,21 \mathrm{~cm}$ (Tableau III). L'analyse de variance, révèle qu'il n'existe aucune différence significative entre les hauteurs moyennes des plants. Les moyennes affectées de la même lettre sont statistiquement identiques pour le test de Tukey au seuil 0,05. La hauteur moyenne des plants exposés à l'ombre de S1 à S3 augmente progressivement et passent de 5,05 $\pm 1,25 \mathrm{~cm}$ à $6,83 \pm 3,29 \mathrm{~cm}$ (Tableau IV). Ensuite de S4 à S7, la croissance en hauteur des plants baisse et passe de $5,084,67 \mathrm{~cm}$ à $0,00 \pm 0,00 \mathrm{~cm}$. À l'opposé, les plants exposés au soleil quant à eux voient leur hauteur moyenne augmentée régulièrement de $\mathrm{S} 1$ à $\mathrm{S} 7$ et passe de $1,59 \pm 0,53 \mathrm{~cm}$ à $3,72 \pm 1,73 \mathrm{~cm}$. L'analyse de variance révèle qu'il existe des différences significatives entre les hauteurs moyennes des plants en considérant l'interaction Semaines-Expositions $(F=51,01 ; p=0,000)$. À cet effet la comparaison des moyennes, montre que de S1 à S4, les plants disposés à l'ombre sont plus hauts. Par contre de S5 à S7, ce sont les plants exposés au soleil qui voient leur hauteur moyenne plus élevée.

Tableau III: Comparaison de la hauteur moyenne des plants en fonction des interactions des paramètres étudiés

\begin{tabular}{ccc}
\hline \multirow{2}{*}{ Substrats/Arrosages } & modalités & hauteur moyenne $(\mathrm{cm})$ \\
& & Sub1Ar1 \\
\cline { 2 - 3 } & Sub1Ar2 & $2,70 \pm 2,97 \mathrm{a}$ \\
\cline { 2 - 3 } & Sub2Ar1 & $3,34 \pm 2,82 \mathrm{a}$ \\
\cline { 2 - 3 } & Sub2Ar2 & $3,36 \pm 2,87 \mathrm{a}$ \\
\hline \multirow{3}{*}{ Expositions/Substrats } & Sub1-omb & $3,36 \pm 3,02 \mathrm{a}$ \\
\cline { 2 - 3 } & Sub1-sol & $3,54 \pm 3,79 \mathrm{a}$ \\
\cline { 2 - 3 } & Sub2-omb & $2,50 \pm 1,43 \mathrm{a}$ \\
\cline { 2 - 3 } & Sub2-sol & $3,89 \pm 3,94 \mathrm{a}$ \\
\cline { 2 - 3 } Expositions/Arrosages & Ar1-omb & $2,83 \pm 1,10 \mathrm{a}$ \\
\cline { 2 - 3 } & Ar1-sol & $3,41 \pm 3,88 \mathrm{a}$ \\
\cline { 2 - 3 } & Ar2-omb & $2,65 \pm 1,36 \mathrm{a}$ \\
\cline { 2 - 3 } & Ar2-sol & $4,01 \pm 3,84 \mathrm{a}$ \\
\hline
\end{tabular}

\section{Quelle information recherchée en procédant de la sorte ??????}

Les moyennes affectées de la même lettre sont statistiquement identiques pour le test de Tukey au seuil 0,05 ( $F=51,01 ; p=0,000)$

Tableau IV: Comparaison de la hauteur moyenne des plants en fonction de l'interaction

(Temps/ Expositions) Pas nécessaire

\begin{tabular}{ccc}
\hline Paramètres & modalités & hauteur moyenne $(\mathrm{cm})$ \\
\hline \multirow{4}{*}{ Temps/Expositions } & S1-omb & $5,05 \pm 1,25 \mathrm{ef}$ \\
\cline { 2 - 3 } & S1-sol & $1,59 \pm 0,53 \mathrm{ab}$ \\
\cline { 2 - 3 } & $\mathrm{S} 2$-omb & $6,44 \pm 1,55 \mathrm{fg}$ \\
\cline { 2 - 3 } & $\mathrm{S} 2$-sol & $2,11 \pm 0,59 \mathrm{bc}$ \\
\cline { 2 - 3 } & $\mathrm{S} 3$-omb & $6,83 \pm 3,29 \mathrm{~g}$ \\
\cline { 2 - 3 } & $\mathrm{S} 3$-sol & $2,46 \pm 0,57 \mathrm{bcd}$ \\
\cline { 2 - 3 } & $\mathrm{S} 4$-omb & $5,08 \pm 4,67 \mathrm{ef}$ \\
\hline
\end{tabular}




\begin{tabular}{cc} 
S4-sol & $2,54 \pm 1,07 \mathrm{bcd}$ \\
\hline S5-omb & $2,16 \pm 4,16 \mathrm{bc}$ \\
\hline S5-sol & $2,90 \pm 1,22 \mathrm{bcd}$ \\
\hline S6-omb & $0,45 \pm 2,09 \mathrm{a}$ \\
\hline S6-sol & $3,27 \pm 1,43 \mathrm{~cd}$ \\
\hline S7-omb & $0,00 \pm 0,00 \mathrm{a}$ \\
\hline S7-sol & $3,72 \pm 1,73 \mathrm{de}$ \\
\hline
\end{tabular}

Incidence de l'interaction des paramètres étudiés sur le nombre moyen de feuilles des plants

Le nombre moyen de feuilles des plants varie de 2,97 $\pm 3,14$ à 6,47 \pm 1,80 respectivement pour les interactions Sub1-omb ; Sub1-sol ; Sub2-omb et Sub2-sol. En considérant à la fois le type de substrat et le mode d'exposition, il n'existe aucune différence significative entre les nombres moyens de feuilles des plants. Le nombre moyen de feuilles des plants exposés à l'ombre de S1 à S7 passe de 4,15 $\pm 1,14$ à $0,00 \pm 0,00$ (Tableau V). En ce qui concerne les plants exposés au soleil il varie de 4,80 $\pm 1,41$ à $7,75 \pm 3,11$. L'analyse de variance montre que les différences des moyennes sont fortement significatives $(F=40,36 ; p=0,000)$. Le test de Tukey au seuil 0,05 révèle que les plants exposés au soleil portent en moyenne plus de

feuilles que les plants exposés à l'ombre. De plus au tout au long des semaines, le nombre moyen de feuilles des plants à l'ombre régresse. Par contre celui des plants exposés au soleil, augmente progressivement jusqu'à S7 où le nombre moyen de feuilles est plus élevé.Les moyennes affectées de la même lettre sont statistiquement identiques pour le test de Tukey au seuil

$$
0,05 ;(F=40,36 ; p=0,000) \text {. }
$$

Tableau V: Tableau de comparaison du nombre moyen de feuilles des plants en fonction de l'interaction Temps /Expositions

\begin{tabular}{ccc}
\hline Paramètres & modalités & Nombre feuilles moyen \\
\cline { 2 - 3 } & S1-omb & $4,15 \pm 1,14 \mathrm{c}$ \\
\cline { 2 - 3 } & S1-sol & $4,80 \pm 1,41 \mathrm{cde}$ \\
\cline { 2 - 3 } & S2-omb & $5,25 \pm 1,33 \mathrm{cdef}$ \\
\cline { 2 - 3 } Temps /Expositions & S2-sol & $5,30 \pm 1,32 \mathrm{cdef}$ \\
\cline { 2 - 3 } & S3-omb & $5,90 \pm 2,63 \mathrm{def}$ \\
\cline { 2 - 3 } & S3-sol & $5,85 \pm 1,31 \mathrm{cdef}$ \\
\cline { 2 - 3 } & S4-omb & $4,45 \pm 4,03 \mathrm{~cd}$ \\
\cline { 2 - 3 } & S4-sol & $5,45 \pm 2,12 \mathrm{cdef}$ \\
\cline { 2 - 3 } & S5-omb & $1,95 \pm 3,78 \mathrm{~b}$ \\
\cline { 2 - 3 } & S5-sol & $0,40 \pm 2,43 \mathrm{efg}$ \\
\cline { 2 - 3 } & S6-somb & $3,27 \pm 1,43 \mathrm{cb}$ \\
\cline { 2 - 3 } & S7-omb & $0,00 \pm 0,00 \mathrm{a}$ \\
\cline { 2 - 3 } & S7-sol & $7,75 \pm 3,11 \mathrm{~g}$
\end{tabular}

\section{L'exploitation des résultats est trop diluée.}




\section{Discussion}

De façon générale, il apparaît que le délai de 11 jours soit celui pour lequel presque toutes les boutures ont bourgeonné. Le délai de bouturage n'a pourtant pas été estimé comme résultat. Cela permet de dire que Thunbergia atacorensis est une espèce à fleur facile à bouturer par rapport à Anogeissus leicarpus (DC) Guill \& Perr. Dans son étude, Dembélé (2012) montre que, Anogeissus leicarpus est une espèce difficile à bouturer. En effet, malgré un traitement avec des hormones particulières, les boutures de Anogeissus leicarpus, mettent 3 semaines pour s'enraciner. Un délai similaire est rapporté sur Ceiba pentandra (Mapongmetsem, 1994) et Lovoa trichilioides (Tchoundjeu et Leakey, 2001). Cette différence sur le délai de bourgeonnement peut s'expliquer par le fait que l'enracinement des espèces tropicales varie dans différents milieux de culture et que cela est très bon dans certains substrats que dans d'autres. Aussi il semblerait, qu'il existe une relation entre la teneur en eau du substrat et celle de la bouture. Par exemple, il est connu que différents substrats affectent l'alimentation en eau des boutures (Grange et Loach, 1983) et ont un effet sur la photosynthèse et la conductance stomatale des boutures (Mesen et al., 1997). Concernant le temps de latence de 3 jours pour les plants au soleil et 5 jours pour les plants à l'ombre cela n'est pas rapporté en résultats, nos résultats montrent que la faible intensité lumineuse ralentit le débourrement des boutures de Thunbergia atacorensis exposées à l'ombre. Cela confirme l'idée de Cornu et Boulay (1986), selon laquelle l'action de la lumière agit sur la vitesse de la reprise végétative des boutures. Toutefois dans notre cas, la faible intensité lumineuse n'empêche pas la reprise végétative de l'espèce cultivée à l'ombre où il y a eu jusqu'à 91p.c. de taux de débourrement. En d'autres termes, comme l'a démontré Hartmann et al. (1997), de façon générale, la photosynthèse n'est pas nécessaire lors de l'enracinement des boutures car elles peuvent utiliser leurs propres réserves pour assurer la reprise végétative ???. Le Taux de survie est nul pour les plants de Thunbergia atacorensis exposés à l'ombre, à partir de la $7^{\text {ème }}$ semaine de mesure. Cela voudrait dire que pour cette espèce, l'intervalle de tolérance pour la lumière est de 6 semaines. Il s'agit, en fait, de la loi de Shelford (Mbakwiravyo, 2009) selon laquelle pour tout facteur écologique, il existe un intervalle de tolérance à l'intérieur duquel tout processus dépendant de ce facteur s'exprime normalement. De part et d'autre des limites de cet intervalle de tolérance, on a des zones létales où la mort de l'organisme résulte d'un défaut ou d'un excès du facteur considéré. Ainsi la faible intensité lumineuse n'empêchant pas le débourrement des boutures, entraine à la longue la mort des plants à l'ombre sachant que le débourrement constitue une étape primordial dans l'enracinement des boutures (Gupta et al., 1997 ; Rai et al.2002). Le taux de survie supérieur à 90.p.c. pour les plants exposés au 
soleil au-delà de la $6^{\text {ème }}$ semaine s'explique donc par la présence de la lumière. Cette différence des taux de survie des plants exposés au soleil et à l'ombre permet de mettre en évidence l'importance de la lumière dans la croissance et le développement des plants (Jacquiot, 1949). Toute fois, selon Kambou (1997), il semblerait que le diamètre des boutures puisse avoir un effet sur le débourrement des boutures donc sur le taux de survie des plants. La croissance en hauteur des plants est dépendante du mode d'exposition. Les plants à l'ombre étant significativement plus hauts que les plants des parcelles exposés au soleil. Cette forte croissance pour les plants des parcelles exposées à l'ombre observée serait due au phénomène d'étiolement. En effet, selon Prat (2008), la plantule, organisme hétérotrophe cultivée en absence ou en quantité insuffisante de lumière, utilise toutes ses ressources pour assurer la croissance dans la tige à la recherche de la lumière. Ce mécanisme observé également chez la tomate, est du au fait qu'en absence de lumière, l'auxine est fortement active et provoque la croissance exacerbée de la tige (Vitre, 2012). À l'opposé, la lumière inhibe l'action de l'auxine et ralentit la croissance des tiges ce qui se traduit par des plants plus courts au niveau des parcelles exposées au soleil. La hauteur moyenne des plants de Thunbergia atacorensis est également évolutive et dépendante du temps. En effet de la $1^{\text {ère }}$ semaine à la $3^{\text {ème }}$ semaine, les plants exposés à l'ombre ont une hauteur moyenne élevée. À l'ombre, ces plants sont donc à la recherche d'une source suffisante de lumière. À partir de la $3^{\text {ème }}$ semaine, les plants atteignent l'intervalle de tolérance à la lumière. Ils s'épuisent car n'arrivant plus à renouveler leur ressources énergétiques par manque d'activité photosynthétique suffisante. On peut donc dire que la 3 ème semaine est la semaine critique lors de la phase de croissance de Thunbergia atacorensis. A partir de cette semaine critique, la mort progressive des plants exposés à l'ombre, se répercute sur la valeur moyenne de la hauteur. C'est ce qui explique, en général, la chute de la hauteur moyenne des plants de la $4{ }^{\text {ème }}$ semaine à la $7^{\text {ème }}$ semaine, à l'ombre. Durant notre étude, les hauteurs moyennes ne diffèrent pas quelque soit le type de substrat ou le mode d'arrosage. Cela s'explique par le fait que tout au long, de l'essai la fréquence d'arrosage et la composition des substrats sont demeurées constantes. Il n'ya eu aucun apport ni modification au niveau du types de substrat ou du mode d'arrosage. La production de feuilles des plants selon nos résultats ne serait influencée ni par la variation du type de substrat, ni par la fréquence d'arrosage, ou même par la combinaison des deux paramètres. Ce constat peut s'expliquer par le fait que, la plante trouve dans ces différentes modalités des conditions favorables à son développement. L'enracinement des espèces tropicales varie dans différents milieux de culture. Cependant il est démontré que leur enracinement est très bon dans certains substrats que dans d'autres. Il reste difficile de déterminer une 
tendance claire. Néanmoins, il semble qu'il existe une relation entre la teneur en eau du substrat et celle de la bouture. Par exemple, il est connu que différents substrats affectent l'alimentation en eau des boutures (Grange \& Loach, 1983) et ont un effet sur la photosynthèse et la conductance stomatale des boutures (Mesen et al., 1997). Par contre, la production de feuilles serait influencée par le mode d'exposition et du temps. En effet, les plants des parcelles exposées au soleil portent plus de feuilles que les plants des parcelles exposées à l'ombre. De plus, de la $1^{\text {ère }}$ semaine à la $7^{\text {ème }}$ semaine, le nombre moyen de feuilles par plant en ce qui concerne ceux exposés au soleil augmente progressivement. La hausse progressive du nombre de feuilles des plants au soleil pourrait s'expliquer par l'action de la lumière qui favorise leur développement par l'activité photosynthétique accrue. À l'opposé, le nombre de feuilles pour les plants des parcelles exposées à l'ombre, régresse pendant la même période. Cette chute progressive du nombre moyen de feuilles des plants à l'ombre est une des conséquences du phénomène de l'étiolement (Renaudin, 2008). En effet, les plants de Thunbergia atacorensis, exposés à l'ombre n'arrivent plus à assurer la croissance et le développement de leurs feuilles. Ainsi cette perte progressive de la capacité à régénérer leurs ressources énergiques par manque de lumière, s'accentue et entraine la chute totale du nombre de feuilles.

\section{Discussion}

De façon générale, il apparaît que le temps moyen de 11 jours soit celui pour lequel presque toutes les boutures ont bourgeonné. Cela permet de dire que Thunbergia atacorensis est une espèce à fleur facile à bouturer. Un délai similaire est rapporté chez Ceiba pentandra (Mapongmetsem, 1994) et Lovoa trichilioides (Tchoundjeu et Leakey, 2001). Ce delai relativement cours de bourgeonnement serait du au fait que les boutures trouvent dans les différents substrats de cultures des conditions favorables. Nos résultats concernant le temps de latence montrant qu'il est de 3 jours pour les plants au soleil et de 5 jours pour les plants à l'ombre, s'explique par le fait que la faible intensité lumineuse ralentit le débourrement des boutures de Thunbergia atacorensis. Cela confirme l'idée de Cornu et Boulay (1986), selon laquelle l'action de la lumière agit sur la vitesse de la reprise végétative des boutures. Toutefois dans notre cas, la faible intensité lumineuse n'empêche pas la reprise végétative de l'espèce cultivée à l'ombre où il y a eu jusqu'à 91p.c. de taux de débourrement. En d'autres termes, comme l'a démontré Hartmann et al. (1997), de façon générale, la photosynthèse n'est pas nécessaire lors de la reprise végétative des boutures car elles peuvent utiliser leurs propres réserves pour assurer la reprise végétative. Le Taux de survie est nul pour les plants de Thunbergia atacorensis exposés à l'ombre, 10 semaines après la mise en terre. Il s'agit, en fait, de la loi de Shelford 
(Mbakwiravyo, 2009) selon laquelle pour tout facteur écologique, il existe un intervalle de tolérance à l'intérieur duquel tout processus dépendant de ce facteur s'exprime normalement. De part et d'autre des limites de cet intervalle de tolérance, on a des zones létales où la mort de l'organisme résulte d'un défaut ou d'un excès du facteur considéré. Ainsi la faible intensité lumineuse n'empêchant pas le débourrement des boutures, entraine à la longue, la mort des plants à l'ombre sachant que le débourrement constitue une étape primordiale dans l'enracinement des boutures (Gupta et al., 1997 ; Rai et al.2002). Le taux de survie supérieur à 90.p.c. pour les plants exposés au soleil s'explique par la présence de la lumière. Cette différence des taux de survie des plants exposés au soleil et à l'ombre permet de mettre en évidence l'importance de la lumière dans la croissance et le développement des plants (Jacquiot, 1949). Toute fois, selon Kambou (1997), il semblerait que le diamètre des boutures puisse avoir un effet sur le débourrement donc sur le taux de survie des plants. La croissance en hauteur des plants à l'ombre étant plus élevée que celle des plants des parcelles exposés au soleil. Cette forte croissance pour les plants des parcelles exposées à l'ombre observée serait due au phénomène de l'étiolement. En effet, selon Prat (2008), la plantule, organisme hétérotrophe cultivée en absence ou en quantité insuffisante de lumière, utilise toutes ses ressources pour assurer la croissance dans la tige à la recherche de la lumière. Ce mécanisme observé également chez la tomate, est du au fait qu'en absence de lumière, l'auxine est fortement active et provoque la croissance exacerbée de la tige (Vitre, 2012). À l'opposé, la lumière inhibe l'action de l'auxine et ralentit la croissance des tiges ce qui se traduit par des plants plus courts au niveau des parcelles exposées au soleil. Durant notre étude, pour un même environnement, les hauteurs moyennes des plantes ne diffèrent pas statistiquement quelque soit le substrat de culture, Cela s'explique par le fait que tout au long, de l'essai la plante trouve dans conditions favorables à son développement. Et de plus l'on pourrait dire qu'il s'agit d'une espèce peu exigeante. Néanmoins en prenant en compte le fait que seuls les plants exposés au soleil survivent au terme de l'essai, le traitement SolSub2Ar1 permet une bonne croissance. Cependant. Il reste difficile de déterminer une tendance très claire au regard de l'analyse statistique. La hauteur moyenne des plants est évolutive tout au long des 7 semaines de mesure et pour les 4 premières semaines elle reste élevée pour les plants à l'ombre que les plants à la lumière. A partir de la 4ème semaine on constate la chute progressive de la hauteur moyenne des plants à l'ombre. En effet, les plants à l'ombre étant à la recherche de la lumière vont subir l'étiolement qui a pour conséquence directe l'allongement de la tige. Les plants atteignent l'intervalle de tolérance à la lumière à la $4^{\text {ème }}$ semaine, elles s'épuisent car n'arrivant plus à renouveler leurs ressources énergétiques par manque d'activité 
photosynthétique suffisante ce qui se répercute sur la valeur moyenne de la hauteur et la mort progressive de celles-ci. Par contre, la production de feuilles serait influencée par le traitement. De plus de la $1^{\text {ère }}$ semaine à la $7^{\text {ème }}$ semaine, le nombre moyen de feuilles par plant en ce qui concerne ceux exposés au soleil augmente progressivement. En effet, les plants des parcelles exposées au soleil portent plus de feuilles que les plants des parcelles exposées à l'ombre. De plus, les plants au niveau SolSub2Ar1 ont un nombre moyen de feuilles beaucoup plus élevé qu'au niveau des autres traitements. La hausse progressive du nombre de feuilles des plants au soleil pourrait s'expliquer par l'action de la lumière qui favorise leur développement par l'activité photosynthétique accrue. À l'opposé, le nombre moyen de feuilles pour les plants exposés à l'ombre, est moins élevé. C'est l'une des conséquences du phénomène de l'étiolement (Renaudin, 2008). En effet, les plants de Thunbergia atacorensis, exposés à l'ombre n'arrivent plus à assurer la croissance et le développement de leurs feuilles.

\section{Pas de conclusion ????? C'est-à-dire que retenir principalement de cette étude ???? \\ Conclusion}

Au total, les résultats de cette étude montrent que la propagation de Thunbergia atacorensis (Acanthaceae) est possible par bouturage. Quelque soit le mode d'exposition ombre ou soleil, plus de $90 \%$ des fragments ont émis des boutures au $11^{\text {ème }}$ jour. Les taux de survie de $0,00 \%$ des plants à l'ombre et de plus de $90 \%$. des plants au soleil observés 10 semaines après la mise en terre, permettent de confirmer que la lumière demeure l'un des facteurs les plus importants pour la culture par bouturage de Thunbergia atacorensis (Acanthaceae). En plus d'un sol de qualité et d'un bon arrosage, la lumière est un facteur indispensable pour la croissance et le développement des plants de Thunbergia atacorensis. Sa quantité suffisante permet d'obtenir des plants plus vigoureux à la longue avec des feuilles mieux développées. À l'opposé, sa faible intensité entraine la mort des plants. Ainsi, au terme de notre essai, nous pouvons dire que le traitement SolSub2Ar1 permet une meilleure croissance des boutures de Thunbergia atacorensis. Aussi, l'absence de floraison constatée qui serait peut être due à l'absence d'apport d'éléments nutritifs au niveau du substrat, ouvre la voie à d'autres études. Pour maîtriser davantage le clonage de cette essence, de nouvelles investigations méritent d'être entreprises pour évaluer la rhizogenèse

Pour la culture de Thunbergia atacorensis, aux horticulteurs, les pepinières peuvent se faire à l'ombre dans les deux premières semaines et par la suite intensifierla luminosité en les rétirant de l'ombrage. En ce qui concerne l'arrosage les résultats ont démontrés que cette plante n'est pas 
exigente en eau et necessite un sol à tendance hydromorphe ici nommé Sub2 provenant de bingerville.

\section{References:}

1. Aké-Assi, E., 1996. Contribution à l'étude des plantes ornementales cultivées dans la région d'Abidjan: Abidjan, Anyama, Dabou, Grand-Bassam (Côte d'Ivoire). Diplôme d'Études Approfondies d'Ecologie Tropicale option VÉGÉTALE, Université de Cocody, $149 \mathrm{p}$.

2. Ake-Assi, E. Koffi, N'G. Tetchi, N. A., 2010. Atlas de la Biodiversité de l'Afrique de l'Ouest, Côte d'Ivoire, TOME III, p324-325.

3. Avit, L. F. JB. Pedia, P. L., Sankaré, Y., 1999. Diversité Biologique de la Côte d'Ivoire-Rapport de Synthèse-Ministère de l'Environnement et de la Forêt, 273p.

4. Bekker, K. Rance W. et Monteuuis, O., 2004. Teak in Tanzania: II. The kilombero Valery

5. teak company. Bois ET Forêts des tropiques 279:pp11-21.

6. Carr, G., 2008. Caractère botanique des Acanthaceae. Mabberley de plant book, Cambridge.

7. University Press, $1021 \mathrm{p}$.

8. Cornu, D. et Boulay, M., 1986. La multiplication végétative : techniques horticole et culture

9. in vitro. Revue Forestière Française, 38 : pp 60-68.

10. Dembélé, C., 2012. Étude préliminaire du potentiel de multiplication par Bouturage de l'Anogeissus leiocarpus (DC) Guil.ET PERR. Influence de l'état physiologique des boutures et régulateurs, Mali, $63 \mathrm{p}$.

11. Dorion, N., 2013. Le bouturage ou comment naissent les racines adventives. Revue de la Société Nationale Horticulteur de France, France, 8p naturel de Côte d'Ivoire. Mémoires ORSTOM n50 Paris(France): pp161-263.

12. Grange R. I. et Loach K. 1983. The water economy of unrooted cuttingd. Journal of Horticultural Science 58:9-17

13. Gupta, V. K. Kumar, R.V. Datta, A. et Solanki, k. R. 1997. Vegetative propagation in Anogeissus pendula. Range Management and Agroforestry $18: 85-90$.

14. Hartmann, T. H. Kester, E. D. Davis, T. F. Jr. Geneve, R. L., 1997. Plant propagation: principles and practices .6è edition, Prentice-Hall inc.Somon ET Schuster, Upper Saddle River, New Jersey 07458, USA, 770p. 
15. Hu, C. et Tsui, H., 2000. Acanthaceae. In Reipubl. Populars sin. 70 : pp 1-309.

16. Jacquiot, 1949. Aperçu sur les problèmes de bouturage en matière forestière. Revue Forestière Français 5:pp 214-223.

17. Kalingani A., Uwamariya A., Larwanou M., 2007. Production de plants agroforestiers,

18. ICRAF Note Technique 1. Nairobi : 47p.

19. Kouadio,Y.J-C, 2009. Étude de la biologie reproductive de Tithonia diversifolia (Hensl)

20. GRAY (Asteraceae) : Espèce non-indigène invasive en Côte d'Ivoire. Mémoire de

21. DEA, UFR BIOSCIENCES-Cocody-Abidjan, 50p.

22. Mapongmetsem P.M. 1994. Phénologie et propagation de quelques essences locales à potenntiel agroforestier en zone forestière. Thèse 3ème cycle. Université de

23. Yaoundé I Cameroun. 172p.

24. MBakwiravyo ,K., 2009. Notes d'Ecologie Générale. Université de conservation de la nature

25. et de développement de Kasugho, 113p.

26. McDade, L. Daniel ,T. et Kiel, C., 2009. Toward a comprehensive understanding of phylogenetic relationships among lineages of Acanthaceae S. L. (Lamiales). Américan Journal of Botany, 95 (9), pp1136-1152.

27. Mesen, J. F., Newton, A.C. et Leakey, R.R.B. 1997. Vegetative propagation of Cordia alliodora (Ruiz et Pavon) Oken : the effects of AIB concentration, propagation medium and cutting origin. Forest Ecology and Management 92:45-54.

28. Myriam, M-B., 2008. Analyse de la variance pour plans à mesures répétées. Cours deMaster1 Psychologie du développement, IRMA, Université LOUIS PASTER, Strasbourg, France ; 88p.

29. Prat, R., 2008. Biologie végétale : Croissance et Développement, $2^{\text {ème }}$ edition $256 \mathrm{p}$.

30. Perraud, A., 1971. Les sols de la Cote d'Ivoire. In le milieu naturel de Cote d'Ivoire Mémoires ORSTOM n50, Paris(France) : pp 269389.

31. Rai, A. K. Solanki, K.R. et Rai, P. 2002. Vegetative propagation in Anogeissus pendula. Range Management and Agroforestry 23 : 166167

32. Renaudin, JP., 1998. UE1. Physiologie et développement des plantes, Université de Bordeaux1. UFR de Sciences Biologiques, $89 \mathrm{p}$. 
33. Riou,G., 1961. Étude de quelques prélèvements de sol à Eleiss (Bingerville) et à Pakidié (Dabou), Mémoires ORSTOM , 28p.

34. Rosenn LP. Denis R., 2004. $A B C$ de la bouture. RUSTICA Edition, 223p.

35. Tchoundjeu Z. et Leakey R.R.B. 2001. Vegetative propagation of Lovoa trichilioides : effects of provenance, substrate, auxins and leaf area. Journal of Tropical forest Science 13(1) :16-129.e

36. Van Der Maessen et Akoegninou A.1999. Flore du Benin, Notes on tropical African plants(2004) 74 :pp 337-345.

37. Vitre A., 2012. La gestion du travail en serre de culture de Tomate, 18p. www.agrieseau.qc.ca/legumesdeserre. Site consulté le 12 février 2013. Date de consultation ???? 